\title{
Early Genomic Detection of Cosmopolitan Genotype of Dengue Virus Serotype 2, Angola, 2018
}

\author{
Sarah C. Hill, Jocelyne Neto de Vasconcelos, \\ Bernardo Gutierrez Granja, Julien Thézé, \\ Domingos Jandondo, Zoraima Neto, \\ Marinela Mirandela, Cruz dos Santos Sebastião, \\ Ana Luísa Micolo Cândido, Carina Clemente, \\ Sara Pereira da Silva, Túlio de Oliveira, \\ Oliver G. Pybus, Nuno R. Faria, \\ Joana Morais Afonso
}

We used portable genome sequencing to investigate reported dengue virus transmission in Angola. Our results show that autochthonous transmission of dengue serotype 2 (cosmopolitan genotype) occurred in January 2018.

$\mathrm{I}^{\mathrm{n}}$ n Africa, the prevalence of disease caused by Aedes mosquito-borne virus infections might be similar to that in the Americas $(1,2)$. However, the transmission and genetic diversity of arthropodborne viruses (arboviruses) in Africa remains poorly understood because of a paucity of systematic surveillance. Moreover, syndromic surveillance might confound symptomatically similar illnesses, and serologic diagnostic tests can be misleading because of cross-reactivity between related circulating flaviviruses (3). Improved viral genomic surveillance can assist in better understanding viral transmission dynamics in Africa.

During 2013, Angola experienced a large dengue outbreak that was concentrated in Luanda Province (4). Cases detected in travelers returning from Angola to other parts of the world showed that the virus rapidly disseminated from Angola to Europe, Asia, and the Americas (5). Although infections were predominantly caused by dengue virus (DENV) serotype 1 (6), all DENV serotypes were reported in returning travelers from Angola (7).

Author affiliations: University of Oxford, Oxford, United Kingdom (S.C. Hill, B. Gutierrez Granja, J. Thézé, O.G. Pybus, N.R. Faria); Instituto Nacional de Investigação em Saúde, Luanda, Angola (J. Neto de Vasconcelos, D. Jandondo, Z. Neto, M. Mirandela, C. dos Santos Sebastião, A.L.M. Cândido, J.M. Afonso); Universidad San Francisco de Quito, Colegio de Ciencias Biológicas y Ambientales, Quito, Ecuador (B. Gutierrez Granja); Cligest, Luanda (C. Clemente, S.P. da Silva); University of KwaZulu-Natal, Durban, South Africa (T. de Oliveira)

DOI: https://doi.org/10.3201/eid2504.180958
Although dengue is probably endemic in Angola, patterns of DENV transmission in the country outside the 2013 epidemic are poorly characterized. The lack of genomic characterization restricts our understanding of DENV diversity within Angola and the frequency and directionality with which DENVs are exchanged with other countries. Although enhanced viral genomic sequencing capacity can improve outbreak detection and response, acquiring this tool remains challenging for many public health laboratories because of the high startup costs of traditional benchtop sequencing (8). Recent technologic advances now permit more affordable sequencing using the MinION portable sequencer (Oxford Nanopore Technologies, https:// nanoporetech.com). We used a combination of portable sequencing and genetic analysis to characterize the causative lineage of a DENV outbreak in Luanda.

\section{The Study}

To investigate the timing and frequency of dengue occurrence in Angola, we conducted rapid diagnostic tests by using the SD Bioline Dengue Duo kit (Alere, https:// www.alere.com) to detect the presence of dengue-specific IgM, IgG, and, since January 2017, nonstructural protein 1 (NS1) (Appendix Figure, https://wwwnc.cdc.gov/EID/ article/25/4/18-0958-App1.pdf). During January 1, 2016May 15, 2018, we collected samples from 6,839 patients (3,276 male, 3,563 female) in central Luanda for whom a physician suspected dengue as the cause of an illness with symptoms consistent with DENV infection. Samples were originally obtained for routine diagnostic purposes from persons visiting local clinics. Thus, we used residual samples without informed consent, with ethics approval from the National Ethical Committee of the Angola Ministry of Health.

We identified 80 DENV NS1-positive cases among the tested samples (Appendix Figure). The first confirmed infections were detected in May 2017, and the number of cases appeared to peak around May 2018 (Figure, panel A).

We tested 153 randomly selected serum samples (IgG, IgM, or NS1-positive) collected during July 21, 2017-January 31,2018 , for DENV RNA by using real-time quantitative reverse transcription PCR (qRT-PCR) at the Instituto Nacional de Investigação em Saúde (INIS) in Luanda, Angola. We used the US Centers for Disease Control and 
Prevention's DENV1-4 Real-Time RT-PCR assay kit on an Applied Biosystems 7500 Fast Real-Time PCR System (https://www.thermofisher.com), according to the manufacturer's instructions (7). Of these 153 samples, 1 (isolate AO-1) yielded a qRT-PCR cycle threshold of 22.5 for DENV serotype 2 (DENV-2). This sample was obtained from a 48-year-old man living in Luanda who visited a clinic on January 18, 2018. The patient reported traveling to Mussulo Island, a resort $30 \mathrm{~km}$ from Luanda, during December 24, 2017-January 2, 2018.

We subjected the qRT-PCR-positive sample to viral genomic amplification and sequencing by using a multiplex PCR primer scheme designed to amplify the entire coding region of DENV-2. We aligned published genomes of nonsylvatic DENV-2 and used them to generate a $90 \%$ consensus sequence that formed the target for primer design. We
A

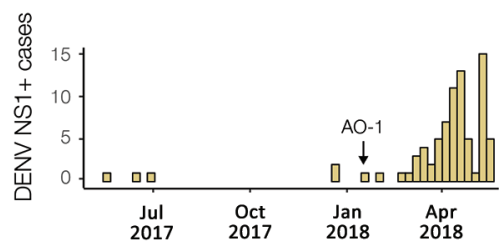

B

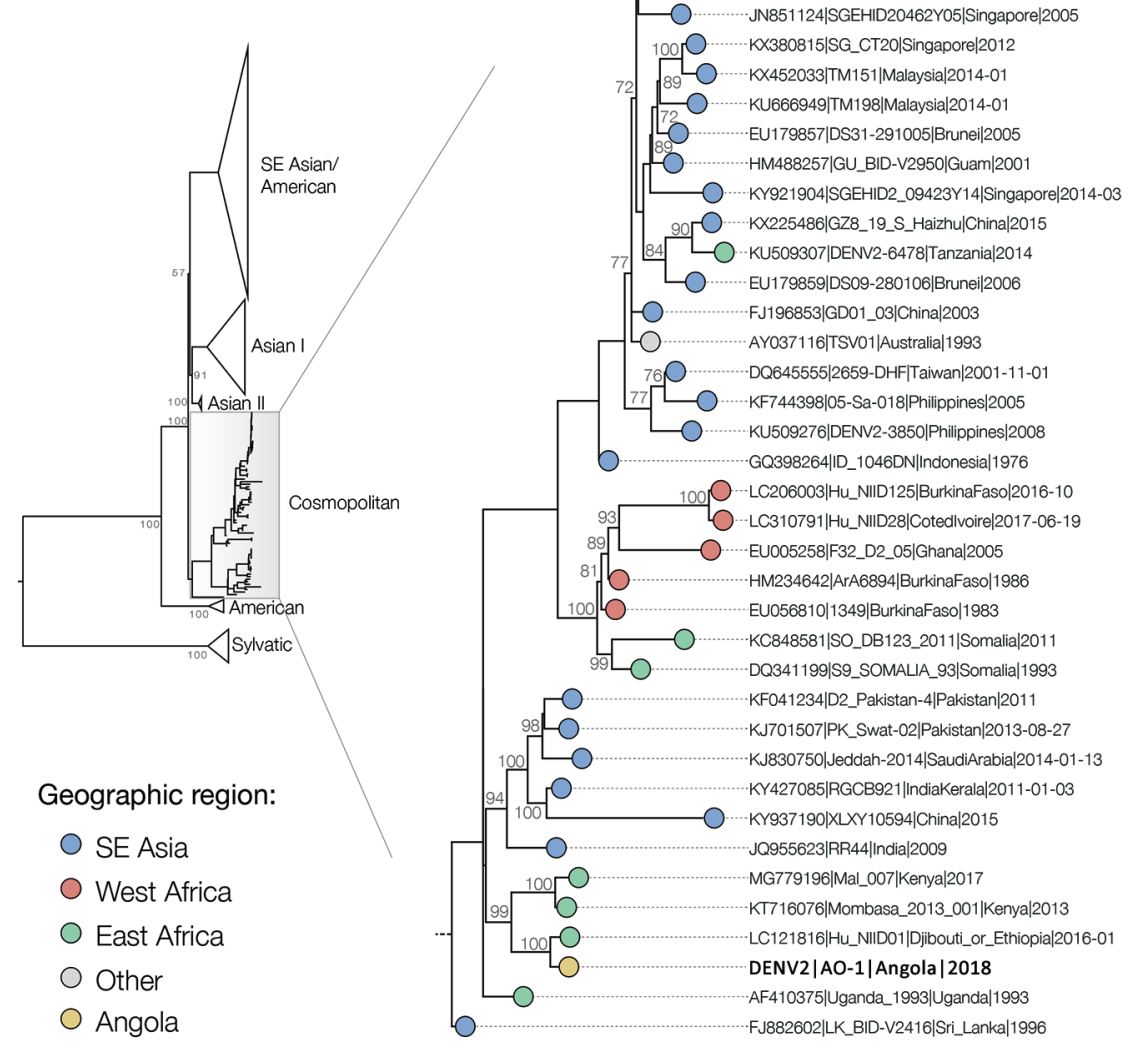

C

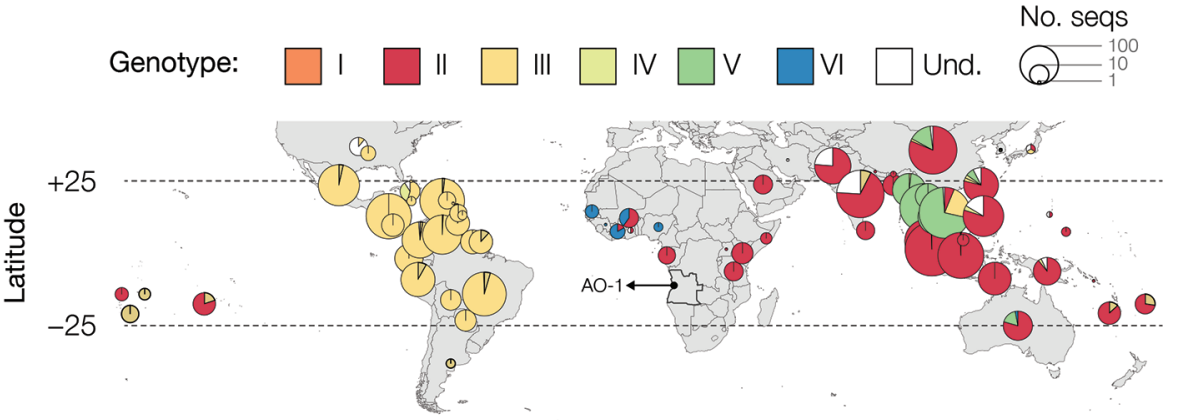

Figure. Investigation of DENV infections in Luanda, Angola, January 1, 2016-May 15, 2018. A) Number of DENV infections (i.e., cases positive for DENV NS1), Luanda, Angola, May 1, 2017-May 15, 2018. B) Midpoint rooted maximum-likelihood phylogeny of DENV-2 whole genomes. Support for branching structure is shown by bootstrap values at nodes. On the right side, the cosmopolitan genotype clade containing the Angola DENV-2 sequence is expanded. Colors indicate geographic location of sampling. The Angola DENV-2 is shown in bold. Support for branching structure is shown by bootstrap values at nodes (bootstrap scores $>70$ shown). C) Geographic distribution of available DENV-2 sequence data (>100 bp). Pie chart radii are log-proportional to the number of sequences available in each country and are colored according to genotype DENV, dengue virus; DENV-2, dengue virus serotype 2; NS1, nonstructural protein 1; SE, Southeast; seq, sequences; und, undefined. 
designed primers that generated overlapping amplicons 980 bp in length with an overlap of $20 \mathrm{bp}$ as previously described (9; Appendix Table). Details of cDNA synthesis, multiplex PCRs, library preparation, sequencing, and generation of consensus sequences are described in the Appendix.

We originally used the $90 \%$ consensus sequence as a reference genome for mapping sequencing reads, but we later refined this reference to a more appropriate reference genome (GenBank accession no. LC121816) by using BLAST (http://www.ncbi.nlm.nih.gov/BLAST) to identify DENV sequences with high identity to the provisionally mapped data. The median sequencing depth was 11,448 reads, and $75 \%$ of the genome had a depth of at least 2,419 reads. In total, we sequenced $96 \%$ of the coding region of DENV-2 (isolate AO-1; GenBank accession no. MH460898). Raw and processed data are available on GitHub (Appendix).

We constructed phylogenetic trees to explore the relationship of the sequenced AO-1 genome to those of other isolates. We retrieved 1,395 DENV-2 genome sequences with associated date and country of collection from GenBank. From this dataset, we generated a subset that included all 35 identified sequences from Africa, 200 globally sampled sequences (randomly sampled from the remaining 1,360 sequences), and the novel AO-1 sequence. We aligned these sequences by using MUSCLE, as implemented in Geneious 9.0.5 (10). We constructed a maximum-likelihood phylogenetic tree by using a general time-reversible model with gamma distributed among site rate heterogeneity (4 categories) in RAXML version 8.2.10 (11). We performed 500 nonparametric bootstrap replicates to evaluate statistical support for phylogenetic nodes. We also constructed a phylogeny that includes additional partial gene sequences (Appendix).

Phylogenetic estimation strongly supports placement of the isolate from Angola in the cosmopolitan genotype of DENV-2 (Figure, panel B). The Angola strain forms part of a well-supported monophyletic clade that comprises genomes sampled in East Africa and is most closely related to DENV isolated from a returning traveler from this region. Viruses from this clade have been present in East Africa since at least 2013 (Figure, panel B).

To explore the global distribution of the DENV-2 cosmopolitan genotype and identify geographic gaps in DENV genomic surveillance that might bias phylogenetic interpretation, we generated maps of the distribution of currently available DENV sequence data. We downloaded from GenBank all DENV sequences $>100$ bp of any serotype with known location of sampling (including those from returning travelers). We genotyped DENV-2 sequences by using the Genome Detective online classification tool (http://www.genomedetective.com). Most sequenced DENVs in Africa belong to DENV-2 (49\%), of which 70\% belong to the cosmopolitan genotype (Figure, panel C). We found that although $16 \%$ of all global clinically apparent dengue infections have been estimated to occur in Africa (2), DENV serotype 1-4 sequences from Africa currently represent $<1 \%$ of the available global DENV sequence data. No data exist from the Democratic Republic of the Congo, which has been epidemiologically linked with Angola during past arbovirus outbreaks (12). Additional data will help to address transmission dynamics of DENV-2 in Angola and identify common routes of virus importation into the country.

\section{Conclusions}

The DENV-2 portable sequencing approach we describe represents a useful tool for genomic characterization and molecular epidemiology of outbreaks in Africa and elsewhere. On the basis of phylogenetic evidence and the geographic distribution of detected genotypes, the DENV-2 cosmopolitan genotype detected in Angola is probably endemic in Africa. The AO-1 genome we analyzed probably represents an early transmission event from an ongoing DENV-2 epidemic in Luanda. Further sequencing of DENV in the region is required to determine whether the cosmopolitan genotype is endemic to Angola or if it represents a more recent introduction from elsewhere (e.g., East Africa or other unsampled locations).

\section{Acknowledgments}

We would like to thank all the patients involved and the staff who assisted with sample collection.

This study was made possible by funding from the Wellcome Trust and Royal Society Sir Henry Dale Fellowship (grant no. 204311/Z/16/Z), the Higher Education Funding Council for England's Global Challenges Research Fund (grant no. 005073), and the John Fell Research Fund (grant no. 005166). Travel to Angola by S.C.H. and N.R.F. was supported by Africa-Oxford Travel Grants (grant nos. AfiOx-48 and AfiOx-60).

This work forms part of the ArboSPREAD project.

\section{About the Author}

Dr. Hill is a postdoctoral researcher at the University of Oxford, United Kingdom. She studies the genomic epidemiology of mosquitoborne viruses, including yellow fever virus, Zika virus, and dengue virus.

\section{References}

1. Gubler DJ. Dengue and dengue hemorrhagic fever. Clin Microbiol Rev. 1998;11:480-96. http://dx.doi.org/10.1128/CMR.11.3.480

2. Bhatt S, Gething PW, Brady OJ, Messina JP, Farlow AW, Moyes CL, et al. The global distribution and burden of dengue. Nature. 2013;496:504-7. http://dx.doi.org/10.1038/nature12060

3. Rabe IB, Staples JE, Villanueva J, Hummel KB, Johnson JA, Rose L, et al. Interim guidance for interpretation of Zika virus 
antibody test results. MMWR Morb Mortal Wkly Rep. 2016; 65:543-6.

4. Centers for Disease Control and Prevention (CDC). Ongoing dengue epidemic - Angola, June 2013. MMWR Morb Mortal Wkly Rep. 2013;62:504-7.

5. Schwartz E, Meltzer E, Mendelson M, Tooke A, Steiner F, Gautret P, et al. Detection on four continents of dengue fever cases related to an ongoing outbreak in Luanda, Angola, March to May 2013. Euro Surveill. 2013;18:20488.

6. Parreira R, Centeno-Lima S, Lopes A, Portugal-Calisto D, Constantino A, Nina J. Dengue virus serotype 4 and chikungunya virus coinfection in a traveller returning from Luanda, Angola, January 2014. Euro Surveill. 2014;19:20730. http://dx.doi.org/ 10.2807/1560-7917.ES2014.19.10.20730

7. Abreu C, Silva-Pinto A, Lazzara D, Sobrinho-Simões J, Guimarães JT, Sarmento A. Imported dengue from 2013 Angola outbreak: not just serotype 1 was detected. J Clin Virol. 2016;79:77-9. http://dx.doi.org/10.1016/j.jcv.2016.04.011

8. Gardy JL, Loman NJ. Towards a genomics-informed, real-time, global pathogen surveillance system. Nat Rev Genet. 2018;19:920. http://dx.doi.org/10.1038/nrg.2017.88

9. Quick J, Grubaugh ND, Pullan ST, Claro IM, Smith AD, Gangavarapu K, et al. Multiplex PCR method for MinION and Illumina sequencing of Zika and other virus genomes directly from clinical samples. Nat Protoc. 2017;12:1261-76. http://dx.doi.org/10.1038/nprot.2017.066

10. Kearse M, Moir R, Wilson A, Stones-Havas S, Cheung M, Sturrock S, et al. Geneious Basic: an integrated and extendable desktop software platform for the organization and analysis of sequence data. Bioinformatics. 2012;28:1647-9. http://dx.doi.org/ 10.1093/bioinformatics/bts 199

11. Stamatakis A. RAxML version 8: a tool for phylogenetic analysis and post-analysis of large phylogenies. Bioinformatics. 2014;30:1312-3. http://dx.doi.org/10.1093/bioinformatics/ btu033

12. Kraemer MUG, Faria NR, Reiner RC Jr, Golding N, Nikolay B, Stasse S, et al. Spread of yellow fever virus outbreak in Angola and the Democratic Republic of the Congo 2015-16: a modelling study. Lancet Infect Dis. 2017;17:330-8. http://dx.doi.org/10.1016/ S1473-3099(16)30513-8

Address for correspondence: Joana M. Afonso, Instituto Nacional de Investigação em Saúde Luanda, Angola; email: jmafonso.7@gmail.com; Nuno R. Faria, University of Oxford, Department of Zoology, Peter Medawar Building, Oxford OX1 3SY, UK; email: nuno.faria@zoo.ox.ac.uk

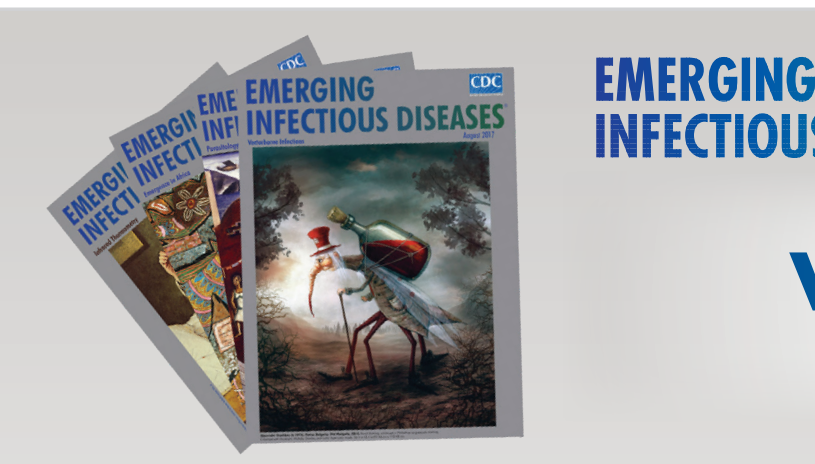

- Added Value of Next-Generation Sequencing for Multilocus Sequence Typing Analysis of a Pneumocystis jirovecii Pneumonia Outbreak

- Bartonella quintana, an Unrecognized Cause of Infective Endocarditis in Children in Ethiopia

- Characteristics of Dysphagia in Infants with Microcephaly Caused by Congenital Zika Virus Infection, Brazil, 2015

- Zika Virus Infection in Patient with No Known Risk Factors, Utah, USA, 2016

- Acute Febrile Illness and Complications Due to Murine Typhus, Texas, USA

- High Infection Rates for Adult Macaques after Intravaginal or Intrarectal Inoculation with Zika Virus

- Lyme Borreliosis in Finland, 1995-2014

- Characterization of Fitzroy River Virus and Serologic Evidence of Human and Animal Infection

- Genomic Characterization of Recrudescent Plasmodium malariae after Treatment with Artemether/Lumefantrine
Vectorborne Infections

- Molecular Characterization of Corynebacterium diphtheriae Outbreak Isolates, South Africa, March-June 2015

- Clinical Laboratory Values as Early Indicators of Ebola Virus Infection in Nonhuman Primates

- Maguari Virus Associated with Human Disease

- Human Infection with Highly Pathogenic Avian Influenza A(H7N9) Virus, China

- Human Metapneumovirus and Other Respiratory Viral Infections during Pregnancy and Birth, Nepal

- Global Spread of Norovirus GII.17 Kawasaki 308, 2014-2016

- Preliminary Epidemiology of Human Infections with Highly Pathogenic Avian Influenza A(H7N9) Virus, China, 2017

- Real-Time Evolution of Zika Virus Disease Outbreak, Roatán, Honduras 